IdeAs

Idées d'Amériques

$5 \mid 2015$

Crise ou transformations du monde du travail dans les Amériques

\title{
Entretien avec Hervé Théry
}

\section{OpenEdition}

\section{Journals}

Édition électronique

URL : https://journals.openedition.org/ideas/811

DOI : 10.4000/ideas. 811

ISSN : 1950-5701

Éditeur

Institut des Amériques

Référence électronique

" Entretien avec Hervé Théry », IdeAs [En ligne], 5 | 2015, mis en ligne le 21 mai 2015, consulté le 19 octobre 2022. URL : http://journals.openedition.org/ideas/811 ; DOI : https://doi.org/10.4000/ideas 811

Ce document a été généré automatiquement le 19 octobre 2022.

\section{(c) $(1) \odot$}

Creative Commons - Attribution - Pas d'Utilisation Commerciale - Pas de Modification 4.0 International - CC BY-NC-ND 4.0

https://creativecommons.org/licenses/by-nc-nd/4.0/ 


\section{Entretien avec Hervé Théry}

1 À l'occasion de l'édition 2015 du Salon du Livre qui s'est déroulée du 20 au 23 mars à Paris et qui mettait à l'honneur le Brésil, nous avons choisi d'interroger Hervé Théry, directeur de recherches au CNRS-Creda, professeur invité à l'Université de São Paulo et codirecteur de la revue Confins.

Hervé Théry était présent à la table ronde « Brésil » le vendredi 20 mars 2015 à $16 \mathrm{~h} 30$.

3 Propos recueillis par Claire Bouffard, secrétaire de rédaction de la revue IdeAs Idees d'Amériques et chargée de mission à l'Institut des Amériques.

C. B. : Le Brésil : un pays émergé ? En termes de relations internationales, quelle place attribueriez-vous à ce pays dans le monde, notamment vis-à-vis du continent africain mais aussi à l'échelle du continent américain ?

H. T. : Ce n'est pas par hasard que j'ai choisi d'intituler mon dernier ouvrage «Brésil, pays émergé » ? Car le Brésil n'apparaît plus aujourd'hui comme un pays émergent. Bien qu'il conserve encore des aspects de pays sous-développé, au même titre que l'Inde ou la Chine, c'est également l'un des plus grands pays du monde de par sa taille, sa population, son PIB, qui figure parmi les dix premiers du globe.

6 Sa situation est toutefois paradoxale puisque le pays ne joue pas un très grand rôle à l'échelle internationale, du moins pas encore, il n'est pas impliqué dans les conflits du Proche-Orient, de la Russie etc. Pourquoi? Tout simplement parce que le pays se considère comme spectateur, il n'est pas impliqué dans les conflits mondiaux. Par exemple, l'ancien ministre des affaires étrangères a déclaré que le Brésil n'avait pas d'ennemis : dans ce cas pourquoi aurait-il donné son avis sur le conflit en Crimée?

7 Il en va de même au sujet du nucléaire iranien : Lula da Silva, ancien président du Brésil a tenté à plusieurs reprises de développer la politique brésilienne en Afrique. Il s'y est rendu 27 fois durant son mandat, a ouvert des ambassades mais cela n'a pas eu grand effet, d'une part parce que de grands pays colonisateurs français et anglais étaient déjà présents, d'autre part car la Chine y intervient de plus en plus. Lula da Silva a réussi à effacer la dette du continent africain envers le Brésil, mais rien de plus.

Quant au continent américain, une politique brésilienne se met en place mais n'est pas encore formalisée. Le MERCOSUR s'est essoufflé et l'UNASUR n'a pas encore donné de résultats. 

Brésil aujourd'hui ? Quelles sont les avancées en termes de recherche scientifique ? Quelques mots sur l'état de la coopération universitaire franco-brésilienne ? Quelles conclusions tirer, par exemple, de votre cartographie des accords CAPES COFECUB ?

13 H. T. : Le monde de la recherche se porte bien au Brésil. Il se développe rapidement. 14 universités ont vu le jour sous Lula da Silva et 4 durant le mandat de Dilma Rousseff. Le nombre de chercheurs n'a cessé d'augmenter ces derniers temps et l'on compte aujourd'hui 10000 docteurs diplômés chaque année. 2014, l'Union internationale des mathématiques a décerné la médaille Fields l'équivalent du prix Nobel pour les mathématiques - au Brésilien Artur Avila lors de l'inauguration du Congrès international des mathématiciens. Rattaché à l'Instituto de Matematica Pura e Aplicada de Rio de Janeiro et au CNRS, ce dernier a été récompensé pour ses profondes contributions à la théorie des systèmes dynamiques.

En termes d'évolution, la coopération universitaire Capes-Cofecub entre la France et le Brésil a dans un premier temps pris la forme d'une assistance française aux universités du Nordeste puis s'est développée par la suite en coopération d'excellence, principalement de l'axe Paris/Rio de Janeiro/São Paulo. Cette coopération d'excellence s'est construite par étapes. D'abord par la coopération de laboratoires de recherche français et brésiliens tous deux très bons. Puis par le biais de collaborations entre des laboratoires français très bons et des laboratoires brésiliens un peu moins bons. Aujourd'hui la tendance se complète par des actions de coopération en recherche entre des laboratoires français " moyens » et de bons laboratoires brésiliens, une supériorité brésilienne qui s'illustre par exemple avec l'étude des zoolithes. Sur ce point, les Brésiliens sont meilleurs que les Français avec leurs découvertes de formations chimiques permettant d'accélérer les catalyses. De la même manière, aujourd'hui les Français n'ont de meilleurs choix que de se déplacer sur place si leur projet de recherche est d'étudier la forêt amazonienne par exemple. 
H. T. : Au sujet des caricatures, je partage l'avis d'Alfredo Valadão (qui a longtemps occupé la chaire Mercosul à Sciences Po), selon lequel le problème du Brésil est que tout ce qui peut être dit sur lui est vrai, des clichés positifs aux plus négatifs, mais que ces dires contradictoires ne reflètent pas l'entière réalité. En effet, le Brésil est par exemple reconnu comme le pays du football; pourtant il a été vaincu de façon presque humiliante lors de la coupe du monde de 2014. Le Brésil est également le pays du carnaval; oui, mais le carnaval ne dure pas toute l'année, seulement quelques jours. Certains aspects seulement de la culture brésilienne sont privilégiés au détriment de la diversité dont témoigne le patrimoine brésilien.

On constate toutefois une évolution positive aujourd'hui dans la façon de décrire le pays. Les journalistes passent plus de temps sur place et donc nuancent davantage leurs propos en ayant une vision plus concrète de la réalité.

D'autre part, le Brésil est un pays qui s'affirme au fur et à mesure. Il a décroché coup sur coup la tenue de deux événements mondiaux majeurs sur son territoire : la Coupe du Monde de Football en 2014 et les Jeux Olympiques d'été en 2016. C'est d'ailleurs pour cette raison qu'Armand Colin m'a sollicité pour publier ce qui est devenu Le Brésil, pays émergé (2014). 2015 étant l'année du Brésil, le Salon du Livre était l'occasion de discuter de mon ouvrage.

\section{B. : Pouvez-vous nous en dire davantage sur le prochain numéro de la revue Confins?}

H. T. : Le numéro 23 de la revue vient d'être mis en ligne. Dans ce numéro, il est intéressant de s'attarder sur l'éditorial qui rend compte de la visibilité de la revue à l'échelle mondiale en présentant une cartographie détaillée. On y aborde également le rôle historique et culturel du Rio São Francisco, ce fleuve qui a fait l'objet d'un colloque international durant l'été 2014 au Brésil.

Quant au dossier thématique du prochain numéro il sera piloté par Olivier Compagnon et traitera de l'immigration au Brésil dans les années 1920.

Confins en chiffres, c'est en moyenne entre 30000 et 40000 visites par mois en 2014, avec une pointe à près de 60000 visites en mars 2015. Et environ huit mois de délai entre l'arrivée d'un manuscrit et sa publication.

Les défis de la revue : Maintenir son statut de revue franco-brésilienne. La commission de classement de l'AERES ne s'étant pas réunie depuis deux ans, la revue perd beaucoup d'auteurs français alors que les auteurs brésiliens, sont nombreux, la revue étant déjà bien classée par la Capes. La revue a donc tendance à devenir davantage brésilienne. 